\title{
INTERSECTION DENSITIES OF NONSTATIONARY POISSON PROCESSES OF HYPERSURFACES
}

\author{
LARS MICHAEL HOFFMANN,${ }^{*}$ Universität Karlsruhe $(T H)$
}

\begin{abstract}
Intersection densities are introduced for a large class of nonstationary Poisson processes of hypersurfaces and inequalities for them are proved. In doing so, similar results from both Wieacker (1986) and Schneider (2003) are summarized in one theorem and the concept of an associated zonoid of a Poisson process of hypersurfaces is generalized to a nonstationary setting.
\end{abstract}

Keywords: Poisson process; intersection process; hypersurface; associated zonoid; Steiner convex set

2000 Mathematics Subject Classification: Primary 60D05

Secondary 52A22; 60G55

\section{Introduction}

Starting with Matheron's introduction of his Steiner convex set in [5], the idea of using an associated convex body to analyse a process of geometric objects has proven itself to be a very fruitful concept. To get an impression of the variety of problems to which this technique has been applied see, for example, the introductions of [11] and [7] or [8, Section 4.5] and the references given therein.

While the case of stationary processes has been treated by Wieacker in a very general way in [9], [10], and [11], some of the results for hyperplane processes have been generalized by Schneider to a nonstationary setting in [7]. In the present paper, we undertake the humble effort to extend a few of these findings.

After introducing some basic notation in Section 2, we define, in Section 3, intersection densities and associated zonoids for certain processes of hypersurfaces, i.e. cylinders with an $\left(\mathcal{H}^{k}, k\right)$-rectifiable set as a basis. Then a connection between intrinsic volumes of associated zonoids and intersection densities is derived. We conclude Section 3 by exploiting this relationship to prove inequalities for intersection densities. The final section is devoted to intersections of these processes with affine subspaces.

We also want to mention that questions regarding measurability and the proofs of some auxiliary results have been moved to the appendix to make this paper more readable.

\section{Preliminaries and basic notation}

Throughout this paper we will work in $d$-dimensional Euclidean space $\mathbb{R}^{d}, d \in \mathbb{N}$, equipped with the canonical structures, $S^{d-1}$ being its unit sphere, $\mathscr{B}\left(\mathbb{R}^{d}\right)$ the Borel $\sigma$-algebra, and $\lambda_{d}$ the

Received 27 October 2006; revision received 22 February 2007.

* Postal address: Institut für Algebra und Geometrie, Universität Karlsruhe (TH), 76128 Karlsruhe, Germany.

Email address: lars.hoffmann@math.uni-karlsruhe.de 
$d$-dimensional Lebesgue measure. More generally, for a topological space $S$, we will always denote the Borel $\sigma$-algebra by $\mathcal{B}(S)$.

Let $k \in\{0, \ldots, d\}$. The $k$-dimensional Hausdorff measure will be denoted by $\mathscr{H}^{k}$ and the $k$-dimensional Lebesgue measure on a $k$-dimensional affine subspace $E$ of $\mathbb{R}^{d}$ by $\lambda_{E}$. Note that any Borel subset of $\mathbb{R}^{d}$ is $\mathscr{H}^{k}$-measurable.

A subset $M$ of $\mathbb{R}^{d}$ is called $k$-rectifiable if and only if either $k=0$ and $M$ is finite or if $k \geq 1$ and there exists a Lipschitz map of some bounded subset of $\mathbb{R}^{k}$ onto $M$. Here, $M$ is called $\left(\mathscr{H}^{k}, k\right)$-rectifiable if and only if $\mathscr{H}^{k}(M)<\infty$ and $\mathscr{H}^{k}$-almost all of $M$ can be covered by some countable family of $k$-rectifiable sets. Finally, $M$ is called $\mathscr{H}^{k}$-rectifiable if $M \cap C$ is $\left(\mathscr{H}^{k}, k\right)$-rectifiable for all compact sets $C \subseteq \mathbb{R}^{d}$.

In the following, the tangential properties of $\left(\mathscr{H}^{k}, k\right)$-rectifiable sets play an important role. Let $M$ be a $\left(\mathscr{H}^{k}, k\right)$-rectifiable subset of $\mathbb{R}^{d}$ with $0<k<d$ and let $\operatorname{Nor}^{k}(M, x)$ denote the cone of all approximate normal vectors of $M$ at $x$. By Theorem 3.2.19 of [2], the latter is a $(\mathrm{d}-k)$-dimensional linear subspace of $\mathbb{R}^{d}$ for $\mathscr{H}^{k}$-almost all $x \in M$. For basic notions from geometric measure theory, we refer the reader to [2].

Let $\mathcal{L}_{k}^{d}$ be the Grassmannian of all $k$-dimensional linear subspaces of $\mathbb{R}^{d}$. For linear subspaces $L_{1}, \ldots, L_{k}$ of $\mathbb{R}^{d}$ with

$$
\operatorname{dim} L_{1}+\cdots+\operatorname{dim} L_{k}=m \leq d,
$$

we choose an orthonormal basis in each space $L_{i}$ (the empty set if $\operatorname{dim} L_{i}=0$ ) and define the determinant $\left[L_{1}, \ldots, L_{k}\right]$ to be the $m$-dimensional volume of the parallelepiped spanned by these $m$ vectors. For a linear subspace $L$, its orthogonal complement will be denoted by $L^{\perp}$ and the orthogonal projection onto $L$ by $p_{L}$.

The space $\mathcal{F}^{\prime}$ of all nonempty closed subsets of $\mathbb{R}^{d}$ will be endowed with the Fell topology and the $\sigma$-algebra $\mathscr{B}\left(\mathcal{F}^{\prime}\right)$. The subspace $\mathcal{K}^{\prime} \subseteq \mathcal{F}^{\prime}$ of all nonempty, compact convex sets (convex bodies) will be equipped with the Hausdorff metric and the $\sigma$-algebra induced by $\mathscr{B}\left(\mathcal{F}^{\prime}\right)$. For all basic notions from convex geometry, we refer the reader to [6]. Finally, let $\mathcal{F}^{(k)} \subseteq \widetilde{F}^{\prime}$ be the subspace of all nonempty, closed $\left(\mathscr{H}^{k}, k\right)$-rectifiable subsets of $\mathbb{R}^{d}$. All required concepts from stochastic geometry (e.g. point processes, intensity measures, or Campbell's theorem) can be found in [8].

\section{Intersection densities and associated zonoids}

Let $l \in\{1, \ldots, d\}$. Throughout this paper, $X_{l}$ will always be a point process on $\mathcal{F}^{\prime}$ with locally finite nontrivial intensity measure $\Theta$ of the form

$$
\Theta(\mathcal{A})=\int_{\mathcal{L}_{d-l}^{d}} \int_{\mathcal{F}^{(l-1)}} \int_{L^{\perp}} \mathbf{1}_{\{\mathcal{A}\}}(M+L+x) f(M+L, x) \lambda_{L^{\perp}}(\mathrm{d} x) \mathrm{P}(L, \mathrm{~d} M) \Phi(\mathrm{d} L),
$$

where $\mathbf{1}_{\{\cdot\}}$ denotes the indicator function and $\mathcal{A} \in \mathscr{B}\left(\mathcal{F}^{\prime}\right)$. Here,

$$
f: \mathcal{F}^{\prime} \times \mathbb{R}^{d} \rightarrow[0, \infty)
$$

denotes a measurable mapping with the additional property that $f(F, \cdot)$ is locally integrable for all $F \in \mathcal{F}^{\prime}$; for $L \in \mathcal{L}_{d-l}^{d}, \mathrm{P}(L, \cdot)$ is a probability measure such that

$$
\mathrm{P}\left(L,\left\{M \in \mathcal{F}^{(l-1)} \mid M \subseteq L^{\perp}\right\}\right)=1
$$


and $L \mapsto \mathrm{P}(L, \mathcal{A})$ is measurable for $\mathcal{A} \in \mathcal{B}\left(\mathcal{F}^{\prime}\right) ; \Phi$ denotes a finite measure on $\mathcal{L}_{d-l}^{d}$. Here, $X_{l}$ can be considered as a process of cylinders, where $\Phi$ describes the distribution of the direction spaces and $\mathrm{P}(L, \cdot)$ the distribution of the basis. Note that $f, \mathrm{P}$, and $\Phi$ are not uniquely determined by $\Theta$.

Let $m \in\{1, \ldots, d\}, n_{0}, \ldots, n_{m} \in\{1, \ldots, d-1\}$, and $M_{0}, \ldots, M_{m} \subseteq \mathbb{R}^{d}$. Furthermore, define

$$
n:=n_{0}+\cdots+n_{m}
$$

We say the pairs $\left(M_{0}, n_{0}\right), \ldots,\left(M_{m}, n_{m}\right)$ satisfy condition $(I)$ if and only if $M_{i}$ is $\mathscr{H}^{n_{i}}$ measurable and $\left(\mathscr{H}^{n_{i}}, n_{i}\right)$-rectifiable for $i \in\{1, \ldots, m\}$ and $M_{0} \times \cdots \times M_{m}$ is $\mathscr{H}^{n}$-measurable and $\left(\mathscr{H}^{n}, n\right)$-rectifiable. By Theorem 4.2 of [4], this is equivalent to the definition of condition $(I)$ in [9]. Examples of pairs of sets satisfying condition $(I)$ can be found in [9, p. 238].

For $i \in\{0, \ldots, m\}$, let $\mu_{i}$ be a measure on $\mathcal{F}^{\left(n_{i}\right)}$. We say the pairs $\left(\mu_{0}, n_{0}\right), \ldots,\left(\mu_{m}, n_{m}\right)$ satisfy condition $(I)$ if and only if the pairs $\left(M_{0}, n_{0}\right), \ldots,\left(M_{m}, n_{m}\right)$ satisfy condition $(I)$ for $\mu_{0} \otimes \cdots \otimes \mu_{m}$-almost all $\left(M_{0}, \ldots, M_{m}\right) \in \mathcal{F}^{\left(n_{0}\right)} \times \cdots \times \mathcal{F}^{\left(n_{m}\right)}$.

Before introducing intersection densities for $X_{l}$, let us prove the following lemma.

Lemma 3.1. Let $j, l \in\{1, \ldots, d\}, L_{1}, \ldots, L_{j} \in \mathcal{L}_{d-l}^{d}$, and $M_{1}, \ldots, M_{j} \in \mathcal{F}^{(l-1)}$ such that

$$
M_{1} \subseteq L_{1}^{\perp}, \ldots, M_{j} \subseteq L_{j}^{\perp} \quad \text { and } \quad\left(M_{1}, l-1\right), \ldots,\left(M_{j}, l-1\right)
$$

satisfy condition (I). Furthermore, let

$$
f_{i}: L_{i}^{\perp} \rightarrow[0, \infty)
$$

be a measurable function for $i \in\{1, \ldots, j\}$ and $B \in \mathcal{B}\left(\mathbb{R}^{d}\right)$ a bounded Borel set. Then

$$
\begin{gathered}
\int_{L_{j}^{\perp}} \cdots \int_{L_{1}^{\perp}} \mathscr{H}^{d-j}\left(B \cap\left(M_{1}+L_{1}+x_{1}\right) \cap \cdots \cap\left(M_{j}+L_{j}+x_{j}\right)\right) f_{1}\left(x_{1}\right) \cdots f_{j}\left(x_{j}\right) \\
\times \lambda_{L_{1}^{\perp}}\left(\mathrm{d} x_{1}\right) \cdots \lambda_{L_{j}^{\perp}}\left(\mathrm{d} x_{j}\right) \\
=\int_{\mathbb{R}^{d}} \mathbf{1}_{\{B\}}(z) \int_{M_{1}} \cdots \int_{M_{j}} f_{1}\left(\left(z-p_{L_{1}}(z)\right)-x_{1}\right) \cdots f_{j}\left(\left(z-p_{L_{j}}(z)\right)-x_{j}\right) \\
\times\left[\operatorname{Nor}^{d-1}\left(M_{1}+L_{1}, x_{1}\right), \ldots, \operatorname{Nor}^{d-1}\left(M_{j}+L_{j}, x_{j}\right)\right] \\
\times \mathscr{H}^{l-1}\left(\mathrm{~d} x_{j}\right) \cdots \mathscr{H}^{l-1}\left(\mathrm{~d} x_{1}\right) \lambda_{d}(\mathrm{~d} z) .
\end{gathered}
$$

Proof. Since $B$ is bounded, there exist cubes

$$
W_{1}, \ldots, W_{j} \in \mathcal{K}^{\prime}
$$

such that $W_{i} \subseteq L_{i}$ and

$$
B \cap\left(M_{i}+L_{i}+x_{i}\right)=B \cap\left(M_{i}+\text { int } W_{i}+x_{i}\right)
$$

for all $x_{i} \in L_{i}^{\perp}$ and $i \in\{1, \ldots, j\}$, where int $W$ denotes the interior of $W$. 
By Fubini's theorem, the following holds:

$$
\begin{aligned}
\int_{\mathbb{R}^{d}} \cdots \int_{\mathbb{R}^{d}} & \mathscr{H}^{d-j}\left(B \cap\left(M_{1}+W_{1}+x_{1}\right) \cap \cdots \cap\left(M_{j}+W_{j}+x_{j}\right)\right) \\
& \times f_{1}\left(x_{1}-p_{L_{1}}\left(x_{1}\right)\right) \cdots f_{j}\left(x_{j}-p_{L_{j}}\left(x_{j}\right)\right) \lambda_{d}\left(\mathrm{~d} x_{1}\right) \cdots \lambda_{d}\left(\mathrm{~d} x_{j}\right) \\
= & \lambda_{L_{1}}\left(W_{1}\right) \cdots \lambda_{L_{j}}\left(W_{j}\right) \\
& \times \int_{L_{j}^{\perp}} \cdots \int_{L_{1}^{\perp}} \mathscr{H}^{d-j}\left(B \cap\left(M_{1}+L_{1}+x_{1}\right) \cap \cdots \cap\left(M_{j}+L_{j}+x_{j}\right)\right) \\
& \times f_{1}\left(x_{1}\right) \cdots f_{j}\left(x_{j}\right) \lambda_{L_{1}^{\perp}}\left(\mathrm{d} x_{1}\right) \cdots \lambda_{L_{j}^{\perp}}\left(\mathrm{d} x_{j}\right) .
\end{aligned}
$$

On the other hand, since $\left(M_{1}+W_{1}, d-1\right), \ldots,\left(M_{j}+W_{j}, d-1\right)$ satisfy the assumptions of Theorem A.1 in the appendix, we obtain

$$
\begin{aligned}
& \int_{\mathbb{R}^{d}} \cdots \int_{\mathbb{R}^{d}} \mathscr{H}^{d-j}\left(B \cap\left(M_{1}+W_{1}+x_{1}\right) \cap \cdots \cap\left(M_{j}+W_{j}+x_{j}\right)\right) \\
& \times f_{1}\left(x_{1}-p_{L_{1}}\left(x_{1}\right)\right) \cdots f_{j}\left(x_{j}-p_{L_{j}}\left(x_{j}\right)\right) \lambda_{d}\left(\mathrm{~d} x_{1}\right) \cdots \lambda_{d}\left(\mathrm{~d} x_{j}\right) \\
& =\int_{\mathbb{R}^{d}} \cdots \int_{\mathbb{R}^{d}} \mathscr{H}^{d-j}\left(B-x_{1} \cap\left(M_{1}+W_{1}\right) \cap\left(M_{2}+W_{2}+x_{2}\right) \cap \cdots \cap\left(M_{j}+W_{j}+x_{j}\right)\right) \\
& \times f_{1}\left(x_{1}-p_{L_{1}}\left(x_{1}\right)\right) f_{2}\left(x_{2}+x_{1}-p_{L_{2}}\left(x_{2}+x_{1}\right)\right) \\
& \times \cdots f_{j}\left(x_{j}+x_{1}-p_{L_{j}}\left(x_{j}+x_{1}\right)\right) \lambda_{d}\left(\mathrm{~d} x_{1}\right) \cdots \lambda_{d}\left(\mathrm{~d} x_{j}\right) \\
& =\int_{\mathbb{R}^{d}} \int_{M_{1}+W_{1}} \cdots \int_{M_{j}+W_{j}} \mathbf{1}_{\left\{B-x_{1}\right\}}\left(t_{1}\right) f_{2}\left(t_{1}-t_{2}+x_{1}-p_{L_{2}}\left(t_{1}-t_{2}+x_{1}\right)\right) \\
& \times \cdots f_{j}\left(t_{1}-t_{j}+x_{1}-p_{L_{j}}\left(t_{1}-t_{j}+x_{1}\right)\right) \\
& \times\left[\operatorname{Nor}^{d-1}\left(M_{1}+W_{1}, t_{1}\right), \ldots, \operatorname{Nor}^{d-1}\left(M_{j}+W_{j}, t_{j}\right)\right] \\
& \times \mathscr{H}^{d-1}\left(\mathrm{~d} t_{j}\right) \cdots \mathscr{H}^{d-1}\left(\mathrm{~d} t_{1}\right) f_{1}\left(x_{1}-p_{L_{1}}\left(x_{1}\right)\right) \lambda_{d}\left(\mathrm{~d} x_{1}\right) \\
& =\lambda_{L_{1}}\left(W_{1}\right) \cdots \lambda_{L_{j}}\left(W_{j}\right) \\
& \times \int_{\mathbb{R}^{d}} \int_{M_{1}} \cdots \int_{M_{j}} \mathbf{1}_{\left\{B-x_{1}\right\}}\left(t_{1}\right) f_{2}\left(t_{1}-t_{2}+x_{1}-p_{L_{2}}\left(t_{1}-t_{2}+x_{1}\right)\right) \\
& \times \cdots f_{j}\left(t_{1}-t_{j}+x_{1}-p_{L_{j}}\left(t_{1}-t_{j}+x_{1}\right)\right) \\
& \times\left[\operatorname{Nor}^{d-1}\left(M_{1}+L_{1}, t_{1}\right), \ldots, \operatorname{Nor}^{d-1}\left(M_{j}+L_{j}, t_{j}\right)\right] \\
& \times \mathscr{H}^{l-1}\left(\mathrm{~d} t_{j}\right) \cdots \mathscr{H}^{l-1}\left(\mathrm{~d} t_{1}\right) f_{1}\left(x_{1}-p_{L_{1}}\left(x_{1}\right)\right) \lambda_{d}\left(\mathrm{~d} x_{1}\right),
\end{aligned}
$$

where the last identity follows from Theorem 3.2.23 of [2]. Combined with another change of variable, this yields the assertion.

Let $j \in\{1, \ldots, d\}$. We introduce a Borel measure $v_{j}$ on $\mathbb{R}^{d}$ by

$$
v_{j}(B):=\mathrm{E} \sum_{\left(M_{1}+L_{1}, \ldots, M_{j}+L_{j}\right) \in\left(X_{l}\right)_{\neq}^{j}} \mathscr{H}^{d-j}\left(B \cap\left(M_{1}+L_{1}\right) \cap \cdots \cap\left(M_{j}+L_{j}\right)\right),
$$

$B \in \mathcal{B}\left(\mathbb{R}^{d}\right)$. Here, $\left(X_{l}\right)_{\neq}^{j}$ denotes the process of all $j$-tupels of $j$ different particles of $X_{l}$. The following result holds. 
Theorem 3.1. Let $j, l \in\{1, \ldots, d\}$ and let $X_{l}$ be a Poisson point process with intensity measure as in (3.1) such that the pairs $\left(\mathrm{P}\left(L_{1}, \cdot\right), l-1\right), \ldots,\left(\mathrm{P}\left(L_{j}, \cdot\right), l-1\right)$ satisfy condition (I) for $\Phi \otimes \cdots \otimes \Phi$-almost all $\left(L_{1}, \ldots, L_{j}\right) \in \mathcal{L}_{d-l}^{d} \times \cdots \times \mathcal{L}_{d-l}^{d}$. Then the measure $v_{j}$ is absolutely continuous with respect to $\lambda_{d}$ with the density given by

$$
\begin{aligned}
& \gamma_{j}(z):=\int_{\mathcal{L}_{d-l}^{d}} \cdots \int_{\mathcal{L}_{d-l}^{d}} \int_{\mathcal{F}^{(l-1)}} \cdots \int_{\mathcal{F}^{(l-1)}} \\
& \times \int_{M_{1}} \cdots \int_{M_{j}}\left.\operatorname{Nor}^{d-1}\left(M_{1}+L_{1}, x_{1}\right), \ldots, \operatorname{Nor}^{d-1}\left(M_{j}+L_{j}, x_{j}\right)\right] \\
& \times f\left(M_{1}+L_{1},\left(z-p_{L_{1}}(z)\right)-x_{1}\right) \\
& \times \cdots f\left(M_{j}+L_{j},\left(z-p_{L_{j}}(z)\right)-x_{j}\right) \\
& \times \mathscr{H}^{l-1}\left(\mathrm{~d} x_{j}\right) \cdots \mathscr{H}^{l-1}\left(\mathrm{~d} x_{1}\right) \mathrm{P}\left(L_{1}, \mathrm{~d} M_{1}\right) \cdots \mathrm{P}\left(L_{j}, \mathrm{~d} M_{j}\right) \\
& \times \Phi\left(\mathrm{d} L_{1}\right) \cdots \Phi\left(\mathrm{d} L_{j}\right), \quad z \in \mathbb{R}^{d} .
\end{aligned}
$$

For $j \in 1, \ldots, d$, we call $\gamma_{j}$ the $j$ th intersection density of $X_{l}$. Sometimes, $\gamma_{1}$ is called the surface area density of $X_{l}$.

Proof of Theorem 3.1. By Lemma A.1, Lemma A.2, and Campbell's and Fubini's theorems, and since $X_{l}$ is Poisson, we have

$$
\begin{aligned}
v_{j}(B)= & \int_{\mathscr{L}_{d-l}^{d}} \cdots \int_{\mathscr{L}_{d-l}^{d}} \\
\times \int_{L_{j}^{\perp}} \cdots \int_{L_{1}^{\perp}} \cdots \int_{\mathcal{F}^{(l-1)}} & \mathcal{H}^{d-j}\left(B \cap\left(M_{1}+L_{1}+x_{1}\right) \cap \cdots \cap\left(M_{j}+L_{j}+x_{j}\right)\right) \\
& \times f\left(M_{1}+L_{1}, x_{1}\right) \cdots f\left(M_{j}+L_{j}, x_{j}\right) \lambda_{L_{1}^{\perp}}\left(\mathrm{d} x_{1}\right) \cdots \lambda_{L_{j}^{\perp}}\left(\mathrm{d} x_{j}\right) \\
& \times \mathrm{P}\left(L_{1}, \mathrm{~d} M_{1}\right) \cdots \mathrm{P}\left(L_{j}, \mathrm{~d} M_{j}\right) \Phi\left(\mathrm{d} L_{1}\right) \cdots \Phi\left(\mathrm{d} L_{j}\right) .
\end{aligned}
$$

First, we assume that $B \in \mathscr{B}\left(\mathbb{R}^{d}\right)$ is bounded. Then, the application of Lemma 3.1 and Fubini's theorem yields

$$
\begin{aligned}
v_{j}(B)=\int_{\mathbb{R}^{d}} \mathbf{1}_{\{B\}}(z) \int_{\mathscr{L}_{d-l}^{d}} \cdots \int_{\mathscr{L}_{d-l}^{d}} \int_{\mathcal{F}^{(l-1)}} \cdots \int_{\mathcal{F}^{(l-1)}} \\
\times \int_{M_{1}} \cdots \int_{M_{j}}\left[\operatorname{Nor}^{d-1}\left(M_{1}+L_{1}, x_{1}\right), \ldots, \operatorname{Nor}^{d-1}\left(M_{j}+L_{j}, x_{j}\right)\right] \\
\times f\left(M_{1}+L_{1},\left(z-p_{L_{1}}(z)\right)-x_{1}\right) \\
\quad \times \cdots f\left(M_{j}+L_{j},\left(z-p_{L_{j}}(z)\right)-x_{j}\right) \mathscr{H}^{l-1}\left(\mathrm{~d} x_{j}\right) \cdots \mathscr{H}^{l-1}\left(\mathrm{~d} x_{1}\right) \\
\quad \times \mathrm{P}\left(L_{1}, \mathrm{~d} M_{1}\right) \cdots \mathrm{P}\left(L_{j}, \mathrm{~d} M_{j}\right) \Phi\left(\mathrm{d} L_{1}\right) \cdots \Phi\left(\mathrm{d} L_{j}\right) \lambda_{d}(\mathrm{~d} z) .
\end{aligned}
$$

By monotone convergence, this is true for arbitrary $B \in \mathcal{B}\left(\mathbb{R}^{d}\right)$.

To introduce a class of zonoids associated with $X_{l}$, we define a Borel measure $\mu_{z}$ on $S^{d-1}$ by

$$
\begin{aligned}
\mu_{z}(A):=\int_{\mathscr{L}_{d-l}^{d}} \int_{\mathcal{F}^{(l-1)}} \int_{M} & \mathcal{H}^{0}\left(S^{d-1} \cap \operatorname{Nor}^{d-1}(M+L, x) \cap A\right) \\
& \times f\left(M+L,\left(z-p_{L}(z)\right)-x\right) \\
& \times \mathscr{H}^{l-1}(\mathrm{~d} x) \mathrm{P}(L, \mathrm{~d} M) \Phi(\mathrm{d} L), \quad A \in \mathcal{B}\left(S^{d-1}\right) .
\end{aligned}
$$


Let $A \in \mathscr{B}\left(S^{d-1}\right)$ and $B \in \mathscr{B}\left(\mathbb{R}^{d}\right)$. Then

$$
\begin{aligned}
\mathrm{E} \sum_{(M+L) \in X_{l}} & \int_{M+L} \mathbf{1}_{\{B\}}(x) \mathscr{H}^{0}\left(S^{d-1} \cap \operatorname{Nor}^{d-1}(M+L, x) \cap A\right) \mathscr{H}^{d-1}(\mathrm{~d} x) \\
= & \int_{\mathscr{L}_{d-l}^{d}} \int_{\mathcal{F}^{(l-1)}} \int_{L^{\perp}} \\
& \times \int_{M+L+z} \mathbf{1}_{\{B\}}(x) \mathscr{H}^{0}\left(S^{d-1} \cap \operatorname{Nor}^{d-1}(M+L+z, x) \cap A\right) \mathscr{H}^{d-1}(\mathrm{~d} x) \\
= & \int_{\mathbb{R}^{d}} \mathbf{1}_{\{B\}}(z) \int_{\mathcal{L}_{d-l}^{d}} \int_{\mathcal{F}^{(l-1)}} \\
& \times \int_{M} \mathscr{H}^{0}\left(S^{d-1} \cap \operatorname{Nor}^{d-1}(M+L, x) \cap A\right) \\
= & \int_{\mathbb{R}^{d}} \mathbf{1}_{\{B\}}(z) \mu_{z}(A) \lambda_{d}(\mathrm{~d} z) .
\end{aligned}
$$

Hence, for $\lambda_{d}$-almost all $z \in \mathbb{R}^{d}$, the measure $\mu_{z}$ is uniquely determined by $\Theta$ (and, thus, does not depend on the choice of $f, \mathrm{P}(L, \cdot)$, and $\Phi)$. Under the additional assumption that

$$
B \mapsto \mathrm{E} \sum_{(M+L) \in X_{l}} \int_{M+L} \mathbf{1}_{\{B\}}(x) \mathscr{H}^{d-1}(\mathrm{~d} x)
$$

is a locally finite Borel measure on $\mathbb{R}^{d}$, a normalization of $\mu_{z}$ could be interpreted as the distribution of the normals of the particles of $X_{l}$ in $z$ whenever $\mu_{z}\left(S^{d-1}\right)>0$. We call $\mu_{z}$ the local mean normal measure of $X_{l}$ at $z$.

By Theorem 2.1 of [3] (and a classical result from convex geometry), for each $z \in \mathbb{R}^{d}$, there exists a unique zonoid $\Pi\left(X_{l}, z\right)$ whose support function is given by

$$
h\left(\Pi\left(X_{l}, z\right), u\right)=\int_{S^{d-1}}|\langle u, v\rangle| \mu_{z}(\mathrm{~d} v), \quad u \in S^{d-1} .
$$

From now on, we refer to $\Pi\left(X_{l}, z\right)$ as the local associated zonoid of $X_{l}$ at $z$.

Let $\operatorname{lin}(x)$ denote the linear hull of a vector $x \in \mathbb{R}^{d}$. From Theorem 2.5 of [3] we obtain, for $j \in\{0, \ldots, d\}$,

$$
\begin{aligned}
& V_{j}\left(\Pi\left(X_{l}, z\right)\right) \\
& =\frac{4^{j}}{j !} \int_{\mathscr{L}^{d} d-l} \int_{\mathcal{F}^{(l-1)}} \cdots \int_{\mathscr{L}_{d-l}^{d}} \int_{\mathcal{F}^{(l-1)}} \\
& \times \int_{M_{1}} \cdots \int_{M_{j}}\left[\operatorname{Nor}^{d-1}\left(M_{1}+L_{1}, x_{1}\right), \ldots, \operatorname{Nor}^{d-1}\left(M_{j}+L_{j}, x_{j}\right)\right] \\
& \times f\left(M_{1}+L_{1},\left(z-p_{L_{1}}(z)\right)-x_{1}\right) \\
& \times \cdots f\left(M_{j}+L_{j},\left(z-p_{L_{j}}(z)\right)-x_{j}\right) \mathscr{H}^{l-1}\left(\mathrm{~d} x_{j}\right) \cdots \mathcal{H}^{l-1}\left(\mathrm{~d} x_{1}\right) \\
& \times
\end{aligned}
$$

We can exploit this last identity to prove the following theorem for the intersection densities. 
Theorem 3.2. Let $j, l \in\{1, \ldots, d\}$ and let $X_{l}$ be a Poisson point process with intensity measure as in (3.1) such that the pairs $\left(\mathrm{P}\left(L_{1}, \cdot\right), l-1\right), \ldots,\left(\mathrm{P}\left(L_{j}, \cdot\right), l-1\right)$ satisfy condition $(I)$ for $\Phi \otimes \cdots \otimes \Phi$-almost all $\left(L_{1}, \ldots, L_{j}\right) \in \mathcal{L}_{d-l}^{d} \times \cdots \times \mathcal{L}_{d-l}^{d}$. For $\lambda_{d}$-almost all $z \in \mathbb{R}^{d}$, we then have

$$
\gamma_{j}(z)=\frac{j !}{4^{j}} V_{j}\left(\Pi\left(X_{l}, z\right)\right)
$$

Moreover, if $j \geq 2$, we have

$$
\gamma_{j}(z) \leq\left(j ! \kappa_{d-1}^{j}\left(\begin{array}{l}
d \\
j
\end{array}\right) / d^{j} \kappa_{d}^{j-1} \kappa_{d-j}\right) \gamma_{1}(z)^{j},
$$

for $\lambda_{d}$-almost all $z \in \mathbb{R}^{d}$, where equality holds if and only if $\Pi\left(X_{l}, z\right)$ is a ball. For $k \in \mathbb{N}, \kappa_{k}$ denotes the volume of the $k$-dimensional unit ball.

Remark. For stationary processes of hypersurfaces, more general results can be found in [10, Section 4] (see e.g. [10, Corollary 2]).

Proof of Theorem 3.2. The first equation follows from Theorem 3.1 and the above formula for $V_{j}(\Pi(X, z))$. By Equation (7.28) of [8, p. 307], we have

$$
\left(\frac{\kappa_{d-1}}{d} V_{1}(K)\right)^{j} \geq \kappa_{d}^{j-1}\left(\kappa_{d-j} /\left(\begin{array}{l}
d \\
j
\end{array}\right)\right) V_{j}(K),
$$

for $K \in \mathcal{K}^{\prime}$ and $j \geq 2$, with equality if and only if $K$ is a ball.

Example. Let $l \in\{1, \ldots, d\}$ and $k \in\{0, \ldots, l\}$. For $L \in \mathcal{L}_{d-l}^{d}$, let $\widetilde{\mathrm{P}}(L, \cdot)$ be a probability measure such that $\widetilde{\mathrm{P}}\left(L,\left\{K \in \mathcal{K}^{\prime} \mid \operatorname{dim} K=k, K \subseteq L^{\perp}\right\}\right)=1$ and $L \mapsto \mathrm{P}(L, \mathcal{A})$ is measurable for $\mathcal{A} \in \mathscr{B}\left(\mathcal{F}^{\prime}\right)$. The dimension of a convex body is defined as the dimension of its affine hull.

Let $X_{l}$ be a Poisson process with intensity measure as in (3.1). Furthermore, we assume that $\mathrm{P}(L, \cdot)$ is the image measure of $\widetilde{\mathrm{P}}(L, \cdot)$ under the mapping $K \mapsto \operatorname{rel}$ bd $K$, where rel bd $K$ denotes the relative boundary of $K$, i.e. the boundary of $K$ in its affine hull. Then $X_{l}$ satisfies the assumptions of Theorem 3.1 and Theorem 3.2.

For $l=1$ and $k=0, X_{l}$ is a Poisson hyperplane process. In this case, Theorem 3.1 and Theorem 3.2 give part of the results of Theorem 2 of [7].

Next, assume that $l=k=d$. By Theorem 2.2.4 of [6], for $\mathscr{H}^{d-1}$-almost all boundary points of a convex body $K$ with nonempty interior, there exists a unique outer normal vector $\sigma_{K}(x)$ of $K$ at $x$. Because $\operatorname{lin}\left(\sigma_{K}(x)\right)=\operatorname{Nor}^{d-1}$ (bd $\left.K, x\right)$, the local mean normal measure $\mu_{z}$ can be written as

$$
\mu_{z}(A)=\int_{\mathcal{K}^{\prime}} \int_{\mathrm{bd} K}\left(\mathbf{1}_{\{A\}}\left(\sigma_{K}(x)\right)+\mathbf{1}_{\{-A\}}\left(\sigma_{K}(x)\right)\right) f(K, z-x) \mathscr{H}^{d-1}(\mathrm{~d} x) \mathrm{P}_{0}(\mathrm{~d} K),
$$

where bd $K$ denotes the boundary of $K$ and $A \in \mathcal{B}\left(S^{d-1}\right)$, with $\mathrm{P}_{0}(\cdot)=\widetilde{\mathrm{P}}(\{0\}, \cdot)$.

For any $K \in \mathcal{K}^{\prime}$, the measure $\mathscr{H}^{d-1}($ bd $K \cap \cdot)$ is equal to the $(d-1)$ th curvature measure of $K$. Thus, by Theorem 4.2 of [1], $B \mapsto \mathrm{E} \sum_{K \in X} \int_{\mathrm{bd} K} \mathbf{1}_{\{B\}}(x) \mathscr{H}^{d-1}(\mathrm{~d} x)$ is a locally finite Borel measure on $\mathbb{R}^{d}$ and, hence, $\mu_{z}$ is a finite measure for $\lambda_{d}$-almost all $z \in \mathbb{R}^{d}$. If we additionally assume that $f \equiv \gamma>0$ is a constant function, Theorem 3.1 and Theorem 3.2 yield Equations (4.54) and (4.55) of [8, p. 164]. 
Furthermore, for all $l \in\{1, \ldots, d\}$ and $k \in\{1, \ldots, l\}$, the above considerations show that the local mean normal measure is of the form

$$
\begin{aligned}
\mu_{z}(A)=\int_{\mathscr{L}_{d-l}^{d}} \int_{\mathcal{K}^{\prime}} \int_{\text {rel bd } K} & \left(\mathbf{1}_{\{A\}}\left(\sigma_{K+L}(x)\right)+\mathbf{1}_{\{-A\}}\left(\sigma_{K+L}(x)\right)\right) \\
& \times f\left(K+L,\left(z-p_{L}(z)\right)-x\right) \\
& \times \mathscr{H}^{l-1}(\mathrm{~d} x) \widetilde{\mathrm{P}}(L, \mathrm{~d} K) \Phi(\mathrm{d} L), \quad A \in \mathscr{B}\left(S^{d-1}\right) .
\end{aligned}
$$

Note that, since $K+L$ has nonempty interior, $\sigma_{K+L}(x)$ can be defined as for convex bodies; $\sigma_{K+L}(x)$ coincides with $\sigma_{K}(x)$ considered in the affine hull of $K$.

\section{Intersections with affine subspaces}

Let $l \in\{1, \ldots, d\}$ and let $X_{l}$ be a point process with intensity measure as in (3.1). In the last section, we introduced associated zonoids for such processes and connected their intrinsic volumes to intersection densities. In this section, we study intersections of $X_{l}$ with affine subspaces. For example, $X_{l}$ induces a (nonstationary) point process onto any affine line and we will show that the intensity function of such a process can be expressed in terms of the support functions of the associated zonoids.

Let $k \in\{1, \ldots, d-1\}, z \in \mathbb{R}^{d}$, and $U \in \mathcal{L}_{k}^{d}$. We want to find an expression for the mean $(k-1)$ th Hausdorff measure of the intersections of the particles of $X_{l}$ with $z+U$ in an arbitrary Borel set $B \subseteq \mathscr{B}(U+z)$. Therefore, we define the following measure:

$$
v_{U+z}(B):=\mathrm{E} \sum_{(M+L) \in X_{l}} \mathscr{H}^{k-1}(B \cap(z+U) \cap(M+L)), \quad B \in \mathscr{B}(U+z) .
$$

Remark. For $k=1, v_{U+z}(B)$ coincides with the mean number of intersection points in $B$ of the line $z+U$ with the particles of $X_{l}$. If $X_{l}$ is stationary and $\lambda_{U+z}(B)=1$, then $v_{U+z}(B)$ is the intensity of the stationary point process induced by $X_{l}$ onto $U+z$ which, by stationarity, is independent of $z$.

The main result of this section is the following theorem.

Theorem 4.1. Let $k \in\{1, \ldots, d-1\}, l \in\{1, \ldots, d\}$, and let $X_{l}$ be a point process with intensity measure as in (3.1). Furthermore, let $U \in \mathcal{L}_{k}^{d}$ and $z \in \mathbb{R}^{d}$. Then $v_{U+z}$ is absolutely continuous with respect to $\lambda_{U+z}$ with the density given by

$$
\gamma_{U+z}(y):=2 \int_{S^{d-1}}\left[\operatorname{lin}(u), U^{\perp}\right] \mu_{y}(\mathrm{~d} u), \quad y \in U+z .
$$

Proof. By Campbell's theorem,

$$
\begin{array}{r}
v_{U+z}(B)=\int_{\mathcal{L}_{d-l}^{d}} \int_{\mathcal{F}^{(l-1)}} \int_{L^{\perp}} \mathscr{H}^{k-1}(B \cap(U+z) \cap(M+L+x)) \\
\times f(M+L, x) \lambda_{L^{\perp}}(\mathrm{d} x) \mathrm{P}(L, \mathrm{~d} M) \Phi(\mathrm{d} L) .
\end{array}
$$


Analogous to the proofs of Lemma 3.1, Lemma A.1, and Lemma A.2, we obtain

$$
\begin{aligned}
v_{U+z}(B)=\int_{z+U} \mathbf{1}_{\{B\}}(y) \int_{\mathcal{L}_{d-l}^{d}} \int_{\mathcal{F}^{(l-1)}} \int_{M} & {\left[\operatorname{Nor}^{d-1}(M+L, x), U^{\perp}\right] } \\
& \times f\left(M+L,\left(y-p_{L}(y)\right)-x\right) \mathcal{H}^{l-1}(\mathrm{~d} x) \\
& \times \mathrm{P}(L, \mathrm{~d} M) \Phi(\mathrm{d} L) \lambda_{U+z}(\mathrm{~d} y) \\
= & \int_{z+U} \mathbf{1}_{\{B\}}(y) 2 \int_{S^{d-1}}\left[\operatorname{lin}(u), U^{\perp}\right] \mu_{y}(\mathrm{~d} u) \lambda_{U+z}(\mathrm{~d} y) .
\end{aligned}
$$

Example. Let $k=1$ and $U:=\{s u \mid s \in \mathbb{R}\}$ for some $u \in S^{d-1}$. In this case, we have

$$
\gamma_{U+z}(z+s u)=2 \int_{S^{d-1}}|\langle v, u\rangle| \mu_{z+s u}(\mathrm{~d} v)=2 h\left(\Pi\left(X_{l}, z+s u\right), u\right),
$$

for $s \in \mathbb{R}$. This is a generalization of Equation (4.50) of [8, p. 159].

\section{Appendix A. Auxiliary and measurability results}

In this section, we collect auxiliary and measurability results needed in Section 3. Respective results implicitly used in Section 4 follow analogously.

The main tool in the proof of Lemma 3.1 is the next result which is a slight generalization of Theorem 3 of [9] and follows directly from the proof of the latter.

Theorem A.1. Letm $\in\{1, \ldots, d\}, n_{0}, \ldots, n_{m} \in\{1, \ldots, d-1\}, B \in \mathcal{B}\left(\mathbb{R}^{d}\right)$, and let $M_{i} \subseteq \mathbb{R}^{d}$ be an $\mathscr{H}^{n_{i}}$-measurable set for $i \in\{0, \ldots, m\}$. Furthermore, define $n:=n_{0}+\cdots+n_{m}$ and let $f: \mathbb{R}^{d} \times \cdots \times \mathbb{R}^{d} \rightarrow[0, \infty)$ be a measurable mapping. We assume that the pairs $\left(M_{0}, n_{0}\right), \ldots,\left(M_{m}, n_{m}\right)$ satisfy condition $(I)$ and that $n \geq m d$. Then

$$
\begin{gathered}
\int_{\mathbb{R}^{d}} \cdots \int_{\mathbb{R}^{d}} \mathcal{H}^{n-m d}\left(B \cap M_{0} \cap\left(M_{1}+x_{1}\right) \cap \cdots \cap\left(M_{m}+x_{m}\right)\right) f\left(x_{1}, \ldots, x_{m}\right) \\
\times \lambda_{d}\left(\mathrm{~d} x_{1}\right) \cdots \lambda_{d}\left(\mathrm{~d} x_{m}\right) \\
=\int_{M_{0}} \cdots \int_{M_{m}} \mathbf{1}_{\{B\}}\left(t_{0}\right) f\left(t_{0}-t_{1}, \ldots, t_{0}-t_{m}\right)\left[\operatorname{Nor}^{n_{0}}\left(M_{0}, t_{0}\right), \ldots, \operatorname{Nor}^{n_{m}}\left(M_{m}, t_{m}\right)\right] \\
\times \mathscr{H}^{n_{m}}\left(\mathrm{~d} t_{m}\right) \cdots \mathscr{H}^{n_{0}}\left(\mathrm{~d} t_{0}\right) .
\end{gathered}
$$

The measurability of the mappings

$$
\left(t_{1}, \ldots, t_{m}\right) \mapsto\left[\operatorname{Nor}^{n_{0}}\left(M_{0}, t_{0}\right), \ldots, \operatorname{Nor}^{n_{m}}\left(M_{m}, t_{m}\right)\right]
$$

and

$$
\left(x_{1}, \ldots, x_{m}\right) \mapsto \mathscr{H}^{n-m d}\left(B \cap M_{0} \cap\left(M_{1}+x_{1}\right) \cap \cdots \cap\left(M_{m}+x_{m}\right)\right)
$$

was proved in [9].

The following two auxiliary results were needed in the proof of Theorem 3.1.

Lemma A.1. Let $k \in\{1, \ldots, d\}, B \in \mathscr{B}\left(\mathbb{R}^{d}\right)$, and

$$
\begin{array}{r}
\mathcal{M}:=\left\{\left(M_{1}^{\prime}, \ldots, M_{k}^{\prime}\right) \in\left(\mathcal{F}^{\prime}\right)^{k} \mid M_{1}^{\prime}, \ldots, M_{k}^{\prime} \text { is }\left(\mathscr{H}^{d-1}, d-1\right)\right. \text {-rectifiable, } \\
\left.M_{1}^{\prime} \cap \ldots \cap M_{k}^{\prime} \text { is a } \mathscr{H}^{d-k} \text {-rectifiable set }\right\} .
\end{array}
$$


Then both $\mathcal{M}$ and the mapping

$$
\left(M_{1}, \ldots, M_{k}\right) \mapsto \mathscr{H}^{d-k}\left(M_{1} \cap \cdots \cap M_{k} \cap B\right) \mathbf{1}_{\{\mathcal{M}\}}\left(M_{1}, \ldots, M_{k}\right)
$$

are measurable.

Proof. By Theorem 2.1.3 of [12] and as, by Theorem 1.1.6 of [8], the mapping $\left(F_{1}, F_{2}\right) \mapsto$ $F_{1} \cap F_{2}, F_{1}, F_{2} \in \mathcal{F}^{\prime}$, is measurable, $\mathcal{M}$ is a measurable set. The result then follows from Theorem 2.1.3, Theorem 2.2.1, and Corollary 2.1.4 of [12].

Lemma A.2. Let $k$ and $\mathcal{M}$ be as in Lemma A.1 and $X_{l}$ as in the assumptions of Theorem 3.1. Then

$$
\mathrm{E} \sum_{\left(M_{1}+L_{1}, \ldots, M_{k}+L_{k}\right) \in\left(X_{l}\right)_{\neq}^{k}} \mathbf{1}_{\left\{\mathcal{M}^{c}\right\}}\left(M_{1}+L_{1}, \ldots, M_{k}+L_{k}\right)=0,
$$

i.e. $\left(M_{1}+L_{1}\right) \cap \cdots \cap\left(M_{k}+L_{k}\right)$ is almost surely an $\mathscr{H}^{d-k}$-rectifiable set for all $\left(M_{1}+L_{1}, \ldots, M_{k}+L_{k}\right) \in\left(X_{l}\right)_{\neq}^{k}$.

Proof. First, let $M_{1}, \ldots, M_{k}, L_{1}, \ldots, L_{k}$ be fixed. From Theorem 3.2.23 of [2] and Theorem 1.4.1 of [12] we obtain that $\left(x_{1}+M_{1}+L_{1}\right) \cap \cdots \cap\left(x_{k}+M_{k}+L_{k}\right)$ is $\mathscr{H}^{d-k}$ rectifiable for $\lambda_{k d}$-almost all $\left(x_{1}, \ldots, x_{k}\right) \in \mathbb{R}^{k d}$.

Since $X_{l}$ is assumed to be Poisson, this, together with Lemma A.1, Campbell's theorem, and Corollary 3.1 .6 of [8], yields

$$
\begin{aligned}
& \mathrm{E} \quad \sum_{\left(M_{1}+L_{1}, \ldots, M_{k}+L_{k}\right) \in\left(X_{l}\right)_{\neq}^{k}} \mathbf{1}_{\left\{\mathcal{M}^{c}\right\}}\left(M_{1}+L_{1}, \ldots, M_{k}+L_{k}\right) \\
& =\int_{\mathcal{L}_{d-l}^{d}} \cdots \int_{\mathcal{L}_{d-l}^{d}} \int_{\mathcal{F}^{(l-1)}} \cdots \int_{\mathcal{F}^{(l-1)}} \\
& \quad \times \int_{L_{k}^{\perp}} \cdots \int_{L_{1}^{\perp}} \mathbf{1}_{\left\{\mathcal{M}^{c}\right\}}\left(x_{1}+M_{1}+L_{1}, \ldots, x_{k}+M_{k}+L_{k}\right) \\
& \times f\left(M_{1}+L_{1}, x_{1}\right) \cdots f\left(M_{k}+L_{k}, x_{k}\right) \lambda_{L_{1}^{\perp}}\left(\mathrm{d} x_{1}\right) \cdots \lambda_{L_{k}^{\perp}}\left(\mathrm{d} x_{k}\right) \\
& \times \mathrm{P}\left(L_{1}, \mathrm{~d} M_{1}\right) \cdots \mathrm{P}\left(L_{k}, \mathrm{~d} M_{k}\right) \Phi\left(\mathrm{d} L_{1}\right) \cdots \Phi\left(\mathrm{d} L_{k}\right)
\end{aligned}
$$

Measurability of the mapping

$$
M+L \mapsto \int_{M+L} \mathbf{1}_{\{B\}}(x) \mathscr{H}^{0}\left(S^{d-1} \cap \operatorname{Nor}^{d-1}(M+L, x) \cap A\right) \mathscr{H}^{d-1}(\mathrm{~d} x)
$$

follows analogously to $[9$, p. 235].

\section{Acknowledgements}

I would like to thank Professor Weil for his help over the last couple of years, all my colleagues for their constant support (especially Dr Steffen Winter for proofreading this paper), and the anonymous referees for their helpful comments. 


\section{References}

[1] Fallert, H. (1996). Quermaßdichten für Punktprozesse konvexer Körper und Boolesche Modelle. Math. Nachr. 181, 165-184.

[2] Federer, H. (1969). Geometric Measure Theory. Springer, Berlin.

[3] Goodey, P. AND WeIL, W. (1993). Zonoids and generalisations. In Handbook of Convex Geometry, eds P. M. Gruber and J. M. Wills, North-Holland, Amsterdam, pp. 1297-1326.

[4] KIÊU, K. (1992). A coarea formula for multiple geometric integrals. Math. Nachr. 156, 75-86.

[5] Matheron, G. (1975). Random Sets and Integral Geometry. John Wiley, New York.

[6] Schneider, R. (1993). Convex Bodies: The Brunn-Minkowski Theory. Cambridge University Press.

[7] Schneider, R. (2003). Nonstationary Poisson hyperplanes and their induced tesselations. Adv. Appl. Prob. 35, 139-158.

[8] SchneIDER, R. AND WeIL, W. (2000). Stochastische Geometrie. Teubner, Stuttgart.

[9] Wieacker, J. A. (1984). Translative Poincaré formulae for Hausdorff rectifiable sets. Geometriae Dedicata 16, 231-248.

[10] Wieacker, J. A. (1986). Intersections of random hypersurfaces and visibility. Prob. Theory Relat. Fields 71, 405-433.

[11] WieAcker, J. A. (1989). Geometric inequalities for random surfaces. Math. Nachr. 142, 73-106.

[12] Z̈̈HLE, M. (1982). Random processes of Hausdorff rectifiable closed sets. Math. Nachr. 108, 49-72. 